\title{
Co-Creation: Human and Al Collaboration in Creative Expression
}

\author{
Sara (Salevati) Feldman \\ Simon Fraser University \\ Canada \\ dr.sara.feldman@gmail.com
}

\begin{abstract}
In recent years, Creative Artificial Intelligence Systems (CAIS) have revolutionised the nature of creative practices. This transformation puts great emphasis on the importance of the human collaborator, the user experience (UX) and the practical applications of CAIS. This paper focuses on understanding how CAIS encourages new modes of creative practice with users through cognitive and emotional UX factors within the domains of creative expressions. It also focuses on co-creativity between human and $\mathrm{Al}$ and what this means for the role of designers/artist today.
\end{abstract}

Creative Artificial Intelligence systems. User experience design. Creative process. Creative expression.

\section{INTRODUCTION}

Computational creativity systems are playing very significant roles in creative activities, such as fine arts, design, and music. Computational creativity, an Artificial Intelligence (AI) subfield, is the study of systems that exhibit behaviour that would be deemed creative in humans. Such computational creativity systems can be used for autonomous creative tasks, such as painting pictures and composing music. Studying computational creativity systems enables us to better understand the human process of creation. Its study also allows us to produce systems for creative people to use, where software becomes a creative collaborator rather than a mere instrument of the artist's creativity. While there is much scholarly discourse on how a Creative Artificial Intelligence System (CAIS) is realised, how it generates, selects and evaluates results, there is less research on the importance of the role of human collaborator, the user experience (UX) and its direct application within a practical environment.

This research is significant as intelligent systems are at time of growth and being utilised in various domains including the art and design field. This adoption requires for new models of creative practice to emerge, where the system acts in collaboration with the users. CAIS systems need to be designed to respond to the emotional and cognitive experience of users, which will lead to the possibility of an act of co-creativity between human and system.
Humans and creative Al can accomplish much more in collaboration with each other, than either could on their own. The human emotion and intuition can train and complement $\mathrm{Al}$ to help lead to brighter outcomes and unexpected possibilities. This impact, however, raises a challenge within the art/design discourse in terms of the creator's role and their authorship.

\section{CAIS}

Systems modelling creativity computationally have gained acceptance in the last decade mainly through their use in artistic and research projects. Computational Creativity Research has most recently been defined by Colton as "the philosophy, science and engineering of computational systems which, by taking on particular responsibilities, exhibit behaviours that unbiased observers would deem to be creative" (Colton \& Wiggins 2012). Several researchers in computational creativity have sought to quantify computational creativity by outlining different dimensions of creativity and proposing schema for evaluating the "level of creativity" of a given system (Ritchie 2007, Colton et al. 2011). Now that more autonomous, generative creative systems have been developed, it is necessary to re-evaluate the role of the human collaborator(s) when designing a creative system for real-world applications in an iterative creative process environment (Shneiderman 2009).

While one area of research in CAIS focuses on the 
total algorithmic automation of the creative process, most research in this area includes some level of human involvement. To make practical applications of CAIS feasible, attention must be paid to the collaborative and creative processes of the users (artists/designers/audiences) who value their experience-developed expertise and intent. Practical systems must be designed to act as support tools for users' cognitive processes as well as providing a poetic and intuitive UX that evokes reflection in the user. CAIS need not only to support exploration, low threshold, high ceiling, wide walls and many paths and styles but also and most importantly - advocate collaboration and open interchange.

Although computational creativity systems cannot directly control a user's creative process, paying close attention to UX and a human's interaction with the system can affect the way decisions are made. Consequently, the user will have an opportunity to gain new perspectives for approaching a creative problem. A user centred approach acts as bridge to create a dialogue between the system and the user; allowing he/she to intuitively, share and exchange knowledge and evolve their creative process in tandem with the system.

An artist painting will exhibit behaviours of both expression and reflection. The painting/design process starts with an initial reflection, and then, through a repeated expressive and reflective process, the artist brings something into being on the canvas. This dialectic between reflection and expression is not an 'on-off' condition but rather a continuous movement between two overlapping poles (DiPaola and Gabora 2007). Our CAIS focuses on this notion of understanding and bringing that cognitive experience into computational systems to support that artistic expression and the magic that happens between the artist and their work. With that emotional expression there is also a shared meaning that goes back and forth, between artist and the audience. The CAIS considered in this paper 'Evolver' system is based on this artistic cognitive model. It explores the generation of new artwork through using genetic algorithms where constraints are expressed as principles, rules and concepts related to the visual organisation of art and design compositions (Dipaola 2014).

\section{EVOLVER}

Evolver is a CAIS, which uses genetic algorithm to produce generative visual design artifacts based on several constraints controlled by the designer/artist. Evolver is born out of an autonomous DarwinsGaze System authored by DiPaola (DiPaola et al. 2013), which explores creativity from theories of cognition, and understand shifts in associative and analytical focus. During the system's successful run at solo shows in noted museums and art galleries, tens of thousands of people believed the artwork was human-created (Figure 1).

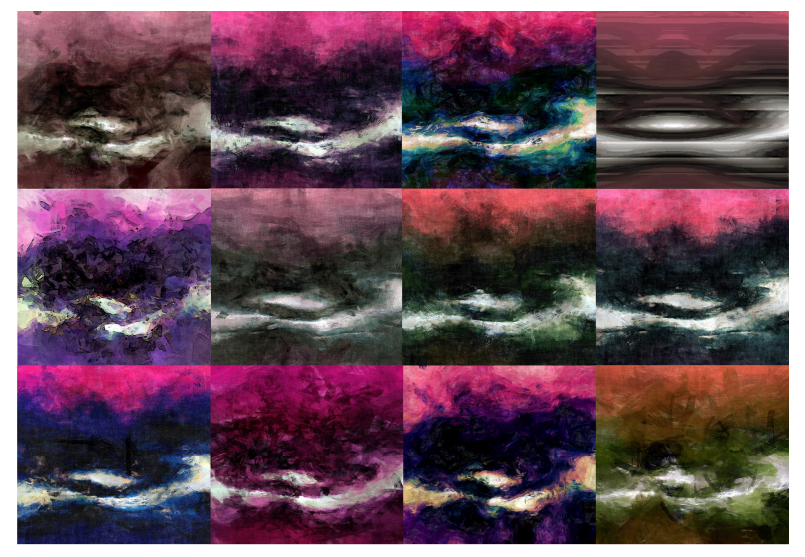

Figure 1: Examples derived from DarwinsGaze

Evolver is a knowledge system comprised of a cognitive model as well as aesthetic reasoning formulated from an artist's creative process. This tool was developed in collaboration by our research team at iVizLab and an industry partner Farmboy Fine Arts (FBFA) (DiPaola et al. 2013). Evolver resulted from an in-depth research and observations done in the aim of supporting a specific design process at FBFA, an international art consultancy firm in Vancouver, BC that designs site- specific art collections for the luxury hotel and corporate sectors. Evolver's main focus was to automate some of the design tasks and restructure the contextual search space within FBFA's creative workflow (DiPaola et al. 2013).

The FBFA workflow includes taking briefs from the hotel interior designers, and based on their extensive photo and graphic design database as source, designs specific art and design objects in a multitude of material (although typically wall hanging) often in unique sizes, shapes and multiples to specifically work with the hotel's (typically their large lobby and restaurants) needs. They do this by incorporating several designers who using digital systems like Adobe Illustrator significantly rework a source design to refit the specifics of space, shape and material.

As researchers we first closely observed FBFA's practical design environment, and documented the designer's already existing process. The FBFA designers' process of creating prototypes for their clients was a multi-step, iterative and somewhat inefficient process, which relied on the designer's 'feel' of the problems and possible solutions. Typically, designers would discuss a project with a client, go to physical boxes or their digital database 
containing image material, locate seed material conforming to the feeling of the project and then manipulate them to fit the design problem in Adobe Illustrator. The designer's manipulation included adjusting size, scale, shape, pattern repetitions, multiples and colour in layers by hand. This process is labour-intensive, and we felt it was a good place for computational support, because the designer had already defined the contextual focus for this problem through their own interpretation of the available options, constraints and aesthetic preferences.

We began by demonstrating to them an interactive version of our Evolver system, which was mocked up on a website, called 'Evolve It' to show what a potentially fully-interactive new system would look like (DiPaola et al. 2013). While the designers were reluctant to give up control of their intuitive, creative process, they readily engaged with Evolve It, once they saw how CAISs could support the restructuring of the designer's contextual space while also reducing the labour- intensive prior process. Adopting CAIS gave designers the ability to creatively engage with the problem at hand. We strove to make the new systems flexible to the unique creative process of each designer (DiPaola et al. 2013).

\subsection{Evolver UX Design}

In an effort to evaluate CAIS within the visual design domain, we explored the use and adaptation of Evolver through UX design strategies. Such strategies include brainstorming to generate versions of original artwork provided by designers, through specific features such as controlling the colour scheme or marrying different artworks together. Evolver also offers some production capabilities by automating repeating tasks, such as cropping for mass quantities of artworks. Designers have traditionally turned to programs such as Adobe Photoshop to complete this task. For ease of use, Evolver incorporates an intuitive graphic user interface (GUI) paired with a flexible internal image representation format (Figure 2) (DiPaola et al. 2013). The designer provides the seed material (Figure 3) and selects preferred results while the system generates a population of artwork candidates and, under user control, uses the CAIS Genetic Algorithm techniques of crossbreeding and mutating the candidates to generate new design products. The designer may select and extract any evolutionary generated contender at any stage of the process for use in other areas or as generative fodder for later projects. System parameters or genes of Evolver include shapes, colours, layers, patterns, symmetries, and canvas dimensions.

Other UX considerations in the design of Evolver include an automatic import tool, which analyses existing images to parse their structure to form initial seed populations for the interactive evolution. This approach serves to bootstrap the evolutionary search with images considered possessive of artistic merit. Source artwork is converted to the SVG (Quint 2003) vector image format, which is a tree-based description of the shapes and curves that comprise a vector-based image. The user interface, by default, depicts a population of eight pieces of generated art. However, our CAIS system has an option to populate more or less depending on the designer's preference. These individuals can be selected to become the parents of the next generation, as is typical in interactive evolution. An added feature, which has proved useful, is the ability to bookmark individual artwork (as in a "favourites" menu). This feature has allowed it to be placed in a collection separate from the evolutionary run while allowing uses to reuse them (and their genetic code) within later new runs. This collection of bookmarked individuals has allowed users to store individuals of interest located during the run while guiding the evolution in a different direction.

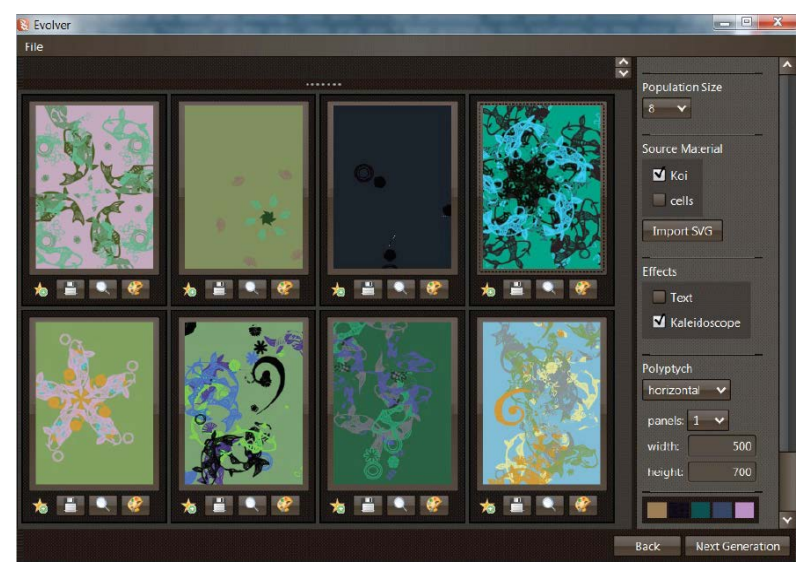

Figure 2: Evolver Interface

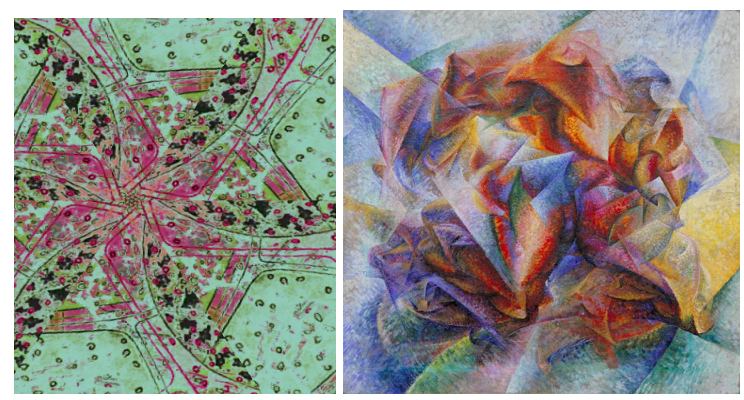

Figure 3: Evolver Outputs

\subsubsection{Evolver UI}

Evolver's cognitive aspect provides designers with a platform to externalise and visualise their ideas. Artwork generated through Evolver can be used for various purposes in all phases of the design process, from conceptual design to presentation. During the early phase of conceptual design, free- 
hand, non-rigid sketching techniques have an important role in the formation of creative ideas as designers externalise their ideas and interact with them spatially and visually (Suwa, Gero and Purcell 1998). Evolver supports flexibility of ideas in this phase by enabling designers to easily produce an extensive range of alternatives. The open-ended nature of the multiple generations produced supports the ambiguous and fuzzy quality of conceptual design as designers discover, frame out early ideas through brainstorming. By producing alternatives for the designers, Evolver helps to relieve their cognitive load. It prevents designers from having to manually manipulate design parameters. At the same time, it does not exclude them from the process to the extent that they cannot use their psychomotor and affective design knowledge.

Evolver is designed to support the shift between contextual and analytical focus by restructuring the contextual space in which users work. Users can choose to relinquish a degree of control while broadening their focus, gaining the ability to be inspired or provoked by the system's novel generations. On the other hand, it is possible to guide successive evolutions in a more deliberate, analytical way, and Evolver's ability to import/export individuals to/from a precisely editable format (SVG - Adobe Illustrator) allows tightly-focused design directions to be pursued. At later stages in the design process, artwork generated through Evolver can be used as prototypes and mockups for clients. It can also be used as a communication tool for presentations at the end of the design process (DiPaola et al. 2013).

\section{EVOLVER STUDY}

This study took place several months after the completion of the design and development of Evolver, with the system still being used and available for real world production at FBFA. We invited a group of ten professional designers to our labs, under controlled study conditions. There we conducted a 45-minute qualitative study that took place in two phases. The first phase involved a uniform re-introduction and re-demonstration of Evolver and its functionalities. The second phase was a session where the designer had the opportunity to re-explore the tool and answer a series of semi-structured interview questions concentrating on the adaptation of Evolver within their current and future work practices. The questions were divided into the following areas:

Introduction. The first set of questions sought the participants' thoughts and opinions on Evolver.

\section{What is your first impression of 'Evolver'?}

2. How and in which stage would you use this tool in your current practice?

Design Process. The second section focused on understanding participants' creative processes and how Evolver could be integrated into their workflow.

3. How does this tool change your design process? Can you provide an existing scenario of your current practice and how you envision Evolver would change that?

4. In which stage of your design process would you most benefit from Evolver? Why?

5. Which features of this tool do you find most interesting? Why?

6. What features would you like to change and/or add in the future? Why?

7. How does it help with the conceptualisation of ideas?

Feedback. The final part of the interview asked participants to comment on their view of computational creativity systems.

8. What do you think of the role of computational creativity systems such as Evolver within the Visual Design domain?

9. Do you have any further comments/suggestions for the future of this research?

\subsection{Data Analysis}

All interviews were audio recorded and transcribed to review data numerous times. For each transcript, we wrote a participant summary based on their exploration of Evolver, as well as detailed interview notes. These transcripts were analysed using axial open and selective coding (Strauss and Corbin 1998) to extract common themes and patterns, which we compared across participants. By assigning keywords such as 'ideation', 'visualisation', 'collaboration' and 'production,' concepts were grouped categorically with relevant quotes and comments from the participants. This process helped to identify overlapping ideas and themes, which will be discussed in detail in the following section. For instance, the transcript tagged with the keywords 'ideation' and 'collaboration' was grouped into the cognitive domain, the 'visualisation' and 'production' texts were categorised into the psychomotor and so forth.

\subsection{Results}

The key findings from this study suggest that the shift of the Visual Design discipline towards a more 
advanced space, with adoption of computational creativity systems not only enhancing designers' skill set, but also encouraging new ways of design. Evolver supports designer's creative process, their workflow and values through a collaborative nature.

The following sections present the findings from our study categorised within 3 main domains based on Bloom's taxonomy: "cognitive, psychomotor and affective" (Bloom 1956). The cognitive domain relies on the tacit and explicit knowledge of the designer. The psychomotor domain encompasses the skills and tools needed to design. Lastly, the affective domain is about the attitude, values, and intent of designers.

\subsubsection{Cognition}

The cognitive domain relies on the knowledge of the designer, that of tacit and explicit. Explicit knowledge can be understood as factual knowledge, which is typically the facts and basic knowledge that are taught in traditional crafts such as medium constraints and typeface recognition (Schön 1983). Additionally, conceptual knowledge helps to shape factual knowledge. In practice, for designers/artists this means that colour theory or Gestalt theory. Tacit knowledge can be linked to procedural knowledge, which is the techniques and judgments exercised during the act of designing, such as choice of colour theme (Schön 1983). However factual and conceptual choice is often unconscious and specific to an individual designer's experience. Design thinking is also tacit and metacognitive knowledge, which is as Lawson describes, the designer's ability to "juggle" multiple perspectives in a problem-solving strategy: the act of framing and re- framing and solution-finding (Lawson 2006).

Design knowledge and reasoning are expressed in the designer's transactions with the artefacts made, the conditions under which they are made, and the manner of making. Design worlds are environments designers inhabit while designing. They contain particular configurations of things, relations and qualities, and they act a place to reserve design knowledge. Each designer has their own unique approach to design. However, with exposure to various solution paths, designers can see how a possible design decision can be matched or mismatched to a situation. In a designer's dialogue with a situation, types of solutions can function to transform the situation and be transformed by it.

From the results of our study it appeared evident that tools such as Evolver assist designers in this process. Evolver facilitates the designer's dialogue with their problem space through the accessibility to alternatives that could inspire various design solutions. Evolver supported the designer's cognitive activity in the early stages of design and the exploration phase by providing an immediate exposure to many possibilities. Designers can then filter these possibilities according to their needs and/or refine and revisit the chosen concepts or ideas.

"Evolver introduces me to design options I never thought of before, it enhances my design thinking and helps me to produce abstract out of the norm ideas." (P1, Interview)

In design, creativity also stems from being selective. The quotes revealed that Evolver helped designers to temporarily step out of their current frame of reference and actively reframe and ask questions. Many of the participants saw the strengths of Evolver within the front-end of their creative process (ideation) in helping to identify the right design questions through discovery of new opportunities. This brings into focus the role of the designer as actor/agent working with cross flows of knowledge, perception and information for filtering, negotiating and mediating design decision-making. The designer is thus simultaneously a participant and observer in an on-going process of collaborative design.

"Evolver provides a spark that inspires new, unexpected ideas, which really helps in creative problem solving, especially when you or your team hits a dead-end." (P2, Interview)

Designers saw Evolver as a creative partner that could suggest alternatives outside of the normal human cognitive capacity:

"[The] Human brain is sometimes limited, I find Evolver to have this unlimited capacity for creativity." (P5, Interview)

\subsubsection{Psychomotor}

The psychomotor domain refers to the skills and tools needed to design. Visualising and sketching are fundamental skills for designers, which enable them to express and communicate their mental models of problem situations, ideas for solutions and rational decision-making in different stages of their design process (Cross 2007). Both visualising and sketching support the fluidity and non-linearity of creative process, through which designers can discover, explore and communicate ideas (Cross 2007). Designers, through the act of sketching can express what they know, what they think and how they imagine. Visualising and sketching allow the designer to "handle different levels of abstraction", "enable identification and recall of relevant knowledge" and "assist problem structuring through solution attempts" (Buchanan 2001). The psychomotor skills also include working with digital programs such as Adobe Illustrator or InDesign as designers partake in intensive training and practice to develop necessary skills to operate these tools. 
Visualisation and sketching also support the fluidity and non-linearity of creative process, through which designers can discover and explore ideas and realise their thinking. The non-permanent nature of sketches allows for experimentation and play throughout the design process. From our study, most designers envisioned integrating Evolver within the early stages of their design process because it provides a platform to externalise and visualise their ideas. They suggested that the exposure to the multiple generations of their idea assisted with the ambiguity of the front-end through discovery, framing out early ideas and brainstorming.

\begin{abstract}
"I would mainly use Evolver as a brainstorming tool within the early stages of my process, as it supports the flexibility of ideas and quickly exposes me to an extensive range of alternatives." (P4, Interview)
\end{abstract}

Evolver facilitated the designer's ability to enter a more intuitive or associative mode of thought by easing some of the effort in manually visualising alternative design concepts:

\begin{abstract}
"Sketching stuff out on paper takes more energy and tweaking - Evolver allows me to visualise easier, have a dialogue and collaborate with the design space." (P3, Interview)
\end{abstract}

The designers in the study described Evolver as an "invisible teammate" who they can collaborate with at any stage of their design process. Evolver could be used flexibly at different stages of the design process to support different tasks and modes of thought, including both the generation and communication of ideas. The participants commented that Evolver acts as a dialogue for designers when communicating their mental models and understanding of a design situation to clients and others involved in the design project.

\subsubsection{Affective}

The affective domain concerns the attitude, values, and intent of designers. These attitudes derive from the internal, personal and emotional experiences of the designer as well as their external influences and context. Designers value empathy and considering their client's wants and needs. Design intent comes with a responsibility that the artistic artefact is never in isolation. Rather it is situated within the need of the individual and the community. Therefore, designers can use their artistic pursuit to provoke an emotional impact through aesthetic expression.

The main issue that CAIS systems such as Evolver raise within the design discourse is that of authorship. Computational creativity systems change the traditional design model and process and this often makes designers feel uneasy about having less control over the originality of ideas produced.
"I think the main issue that comes to mind in designing with computational creativity systems, is the ownership of the work. It's a grey area, am I the creator or the system? I could see how some traditional designers would be resistant to give up that control. However, design nowadays has become more of a collaborative and participatory process, you do not design in isolation, you co-create with your team, clients and stakeholders. Evolver is one of those collaborators." (P4, Interview)

As exemplified by this quote, most the designers who participated in the study commented on Evolver's inability (and CAIS in general) to incorporate the richness of their emotional intent, the expression of a certain mood they wanted their designs to provoke and communicate. For example:

\begin{abstract}
"I create mood boards for each design project based on client brief, searching images in Google, magazines to express a certain emotion I want my design to express. For example, I had a client that wanted an ad campaign that presented serenity and tranquillity in communicating their message. It would be nice if there was a way to control that aspect in Evolver." (P6, Interview)
\end{abstract}

\subsubsection{Findings}

This study revealed that CAIS such as Evolver can act collaboratively to assist designers in their creative process. These creative systems need to be augmented in designer's work process, as they see fit, rather than demanding designers to adjust their process posed by the technology. These tools can facilitate the dialogue designers have with their problem space through the accessibility to alternatives that could inspire various design solutions. Artwork generated through Evolver can be used for different purposes in different phases of the design process. They can be used as brainstorming tools, usually in the front-end of design process; as mock-ups for clients and prototyping towards the end of design process; and also as a communication tool such as presentations. Evolver is an activity among designers that facilitates collaboration.

A fascinating discovery from this study was the notion of artistic pursuit, a sense of communication, articulation, and expression of emotion. The incorporation of mood/affect into the design of computational creativity systems is the outmost important factor of CAIS. Shared authorship, coauthorship and design can be explained as collaboration between designers and computational creativity systems. This participatory and inclusive approach is an important design consideration 
because such systems should be aware of the creative user's expertise, emotion and experiences in order to create personal meaning. In the end, this generates results in a richer creative experience and innovative output.

This inquiry aims to address and bridge the gap between the growing emotional and expert knowledge of designers who are searching for democratic participation in the dissemination of their creative expression. This can be seen as one of the more important roles for computational creativity systems.

Many study responses revealed the attitude shift of visual designers towards the new model of design, in which their role changes from sole creators to editors and collaborators. Artists and designers have become more receptive to systems such as Evolver, as they are not meant to replace designers and to fully automate the creative process. Rather to promote new ways of design and take designer's abilities to the next level by providing efficiency, encouraging more 'aha' moments and lead to unexpected results.

Another design consideration that was evident from the study and observations of designers exploring Evolver was the change to the shift away from the linearity of the program. Most designers expressed how they would like to visually see the evolvement of the genetic seed file within the various spaces such as colour and shape. They want to see how Al truly designs, not be a black box. Designer want to keep track of evolutionary generation within each category and have more control in selecting and cross breeding (marrying) different artworks based on those separations. This would also help with archiving and saving the various generations. Another suggestion would be that within each generation an out of the norm choice is produced (i.e. from a different part of the genetic search space), to provide designers with a novel option that sparks an inspiration. This keeps designers engaged in the program as their exposure to an unexpected solution path can trigger more 'aha' moments and keep them from being stuck and remaining in the same frame of reference.

The main limitation of this study is the time and amount of exposure the designers had to the Evolver system. Long-term access to the tool and a complete observation of its use with an actual design project will offer a more in-depth understanding of its effect and influence on designer's current processes. However, this study provided an initial understanding and identified opportunities by highlighting areas in which computational creativity systems can leverage and enhance the designer's process. While there were only 10 designers represented, the responses were regarded as coherent and comprehensive in relation to the theoretical framework of creativity.

\section{CONCLUSION}

This research offers exciting opportunities for designing interactions with future Al systems, to help augment our creativity and become reactive to our emotional experiences. This allows for a collaboration between human and $\mathrm{Al}$ and realisation of something novel that the neither could do alone.

We believe the traditional role of a designer/artist is here to stay and will not be replaced by such tools, however designers now have the opportunity to explore and balance traditional and new mediums to shape and evolve their design process. This will only increase appreciation and demand in this domain. This leap towards a new model of design changes the role of designers as sole creators to editors and orchestrators. Al alone is not cable of creating novel ideas, they produce acceptable work but not exceptional. Human interaction is necessary in evaluating the value of the produced work within a given context. When human and $\mathrm{Al}$ join forces, it can lead to surprising, innovative and unimaginable work.

CAIS use algorithmic techniques to provide designers with a more abstracted interface for creation. By allowing users to give sufficient highlevel direction, goals and constraints, CAIS can produce millions of variations of an artwork. Designers get to become curators, picking their favourite or keep re-mixing until they get closer to a great design quickly and easily. This allows for efficiency and an increase in productivity by being able to explore many alternative directions in a fraction of the time.

CAIS may be more efficient and effective at automation and delivery but they lack to understand the entire problem, such as context, culture, emotion and empathy. CAIS tends to struggle with emotional awareness and overall social intelligence. This also extends to understanding of human intent, motivations and actions.

We believe, CAIS should look at the combination of cognitive and affective creativity and the experience of its users. They complement each other and make it possible to bring in different aspects of creativity, in different phases of collaborative creative practice. The characteristic of the cognitive creativity knowledge - the inside turns and becomes the outside - the UX through a seamless dialogue. A UX perspective in designing CAIS, relies on the understanding of creative 
experience as co-creation, where distinctions between tangibles and intangibles are periphery. Instead, the focus is transferred to complex relations, interactions and human collaborators, which is highly empathic.

We want to continue designing CAIS that its sole focus is not to produce a novel work but to learn and create models based our individual style, challenge itself, generate new creative questions and solutions. This is an exciting opportunity for the creative industry. The future CAIS systems to not only enhance our creativity but understand our experiences, influences, inspirations and be able to critique our work and suggest improvements.

\section{FUTURE DIRECTION}

Until recently, the relationship between the discourses of computational creativity and UX design has been quite distant. This is mainly due to the autonomous nature of computational creativity systems that are designed to mimic human-like creative process cognitively without human input. The applicability of CAIS within various domains however, is drawing attention and showing an increase awareness of the importance of practical and societal aspects of computational creativity. This suggests opening up to larger complexity and an awareness of relations between collaborators and intelligent systems.

In conclusion, this research deepens the knowledge and understanding, thereby awareness of the significance in the integration of affect and UX concepts within the computational creativity field. It will also inform the future design, interdisciplinary and possible practical applications of CAIS.

\section{REFERENCES}

Bloom, B. S., Engelhart, M. D., Furst, E. J., Hill, W. H., and Krathwohl, D. R. (1956) The Taxonomy of Educational Objectives, The Classification of Educational Goals, Handbook I: Cognitive Domain. New York: Longman.

Buchanan, R. (2001) Design and the New Rhetoric: Productive Arts in the Philosophy of Culture. Philosophy and Rhetoric, 34 (3), pp. 183-206.

Colton, S., Charnley, J., and Pease, A. (2011) Computational creativity theory: The face and idea descriptive models. In Proceedings of the International Conference on Computational Creativity.

Colton, S. and Wiggins, G. (2012) Computational
Creativity: The Final Frontier? In: L. de Raedt, C. Bessiere, D. Dubois, \& P. Doherty (eds.), Proceedings of the $20^{\text {th }}$ European Conference on Artificial Intelligence. 242 Amsterdam: IOS Press, pp. 21-26.

Cross, N. (2007) Designerly Ways of Knowing. London: Springer.

DiPaola, S. (2014) Using a Contextual Focus Model for an Automatic Creativity Algorithm to Generate Art Work, Procedia Computer Science. Special Issue: Bio Inspired Cognitive Architectures, 41, pp. 212-219.

DiPaola, S. and Gabora, L. (2007) Incorporating characteristics of human creativity into an evolutionary art algorithm. In D. Thierens (ed.), Proceedings of the Genetic and Evolution Computing Conference, pp. 2442-2449. July 7-11, University College London, UK.

DiPaola, S., McCaig, R., Carson K., Salevati, S., and Sorenson, N., (2013) Adaptation of an Autonomous Creative Evolutionary System for Real-World Design Application Based on Creative Cognition. International Conference on Computational Creativity (ICCC 2013), Sydney, Australia, pp. 40-47.

Lawson, B. (2006) How Designers Think: The design process demystified. New York, New York: Architectural Press.

Quint, A. (2003) Scalable Vector Graphics. IEEE Multimedia, 10(3), pp. 99-102.

Ritchie, G. (2007) Some empirical criteria for attributing creativity to a computer program. Minds and Machines, 17.

Schön, D. (1983) The Reflective Practitioner. New York: Basic Books.

Shneiderman, B. (2009) Creativity support tools: A grand challenge for $\mathrm{HCl}$ researchers. In $\mathrm{M}$. Redondo, C. Bravo, \& M. Ortega (eds.), Engineering the User Interface, pp. 1-9. SpringerVerlag London.

Strauss, A., and Corbin, J. (1998) Basics of Qualitative Research, 2nd ed. Thousand Oaks, CA: Sage.

Suwa, M., Gero, J. S., and Purcell, T. (1998) The roles of sketches in early conceptual design processes. In Proceedings of Twentieth Annual Meeting of the Cognitive Science Society, pp. 1043-1048. 